\title{
Circuit Theory Analysis of Acoustically Coupled Rooms
}

\author{
Análisis circuital de espacios acústicamente acoplados
}

\author{
Federico Miyara \\ Laboratorio de Acústica y Electroacústica, Facultad de Ciencias Exactas, Ingeniería y Agrimensura (FCEIA), \\ Universidad Nacional de Rosario. \\ Riobamba 245 bis, Rosario, Argentina \\ fmiyaralfceia.unr.edu.ar \\ Recibido: 02/12/18; Aceptado: 12/02/19
}

\begin{abstract}
A circuit theory approach is presented for the analysis of acoustically coupled spaces. It is based on energy flow and balance with a similar analogy to the one used for solving dynamic thermal systems, where acoustic power is analog to electric current and acoustic energy density is analog to voltage. Room volume is equivalent to capacitor and sound absorption is equivalent to a resistor. From these analogies and Laplace-transformed models it is possible to get stationary or transient response solutions.
\end{abstract}

Keywords: coupled rooms; circuit model; analogies

Resumen- Se propone un enfoque circuital basado en flujos de energía para el análisis de espacios acústicamente acoplados. En el mismo se establece una analogía similar a la que se utiliza en el estudio de sistemas térmicos dinámicos en el cual la potencia acústica es análoga a la corriente eléctrica y la densidad de energía acústica es análoga a la tensión. Los volúmenes son equivalentes a capacitores y la absorción sonora es asimilable a una resistencia. A partir de estas analogías se pueden plantear ecuaciones diferenciales y modelos en transformada de Laplace y obtener tanto soluciones estacionarias o de régimen permanente como transitorias.

Palabras clave: recintos acoplados; modelo circuital; analogías

\section{INTRODUCTION}

Physical systems can be represented by different formal frameworks. Some of them are block diagrams, graph theory [1] and bond graphs [2]. Often the choice of one or another depends on the nature of the process and on the kind of the desired results. In the case of electric networks, circuit theory, based on Ohm's and Kirchhoff's laws, provides an adequate approach because of the simplicity of the graphical representation akin to the actual topology of the physical system, and the availability of methods to write down the equations by simple inspection. The use of the Laplace transform allows to easily analyze circuits with dynamic components such as capacitors and inductors.

The degree of maturity of circuit theory and the familiarity with its techniques reached by electrical engineers and other practitioners has prompted the extension of its principles to other specialties such as hydraulics, mechanics and acoustics [3]. The general approach is to select two relevant variables that can be considered analogous to voltage and electric current and identify physical subprocesses where these variables are linked. In general, but not always, the variables are chosen so that their product has dimension of power. This allows, by means of conservative transducers, the transition from a physical domain to another, such as is the case of electroacoustic equivalent circuits [3].

For instance, in the case of mechanical circuits, the variables are force and velocity, and in the case of acoustic or hydraulic systems, pressure and flow rate (or volume velocity). In general there is a flow type variable and a force type variable, which are the analogs to current and voltage respectively.

Our purpose is to introduce an equivalent-circuit approach applicable to the analysis of acoustically coupled rooms in both static and dynamic conditions.

\section{ANALOGIES}

In the case of acoustically coupled spaces which we shall be dealing with, we will take the acoustic power as the analog of current. Should we stick to the rule that the product of the two variables has dimension of power, the second variable should be non-dimensional, which is not convenient.

We have the precedent of thermal circuits, in which the relevant circuit variables are power and temperature [4], whose product has not dimension of power. They are often used to estimate the working temperature of electronic components (Fig. 1).

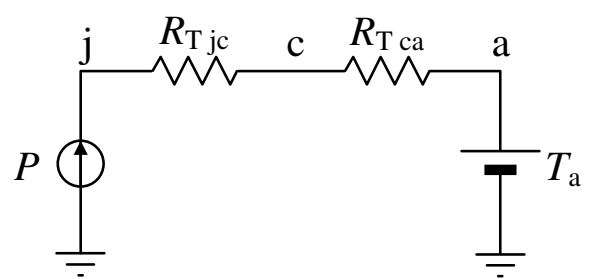

Fig. 1. An example of thermal circuit in which there is a power source (the junction), the thermal resistance between the junction and the case, $R_{\mathrm{T}}$ c, the thermal resistance between the case and the environment, $R_{\mathrm{T} \mathrm{jc}}$, and a fixed ambient temperature, $T_{\mathrm{a}}$.

In order to select the force variable it will be useful to analyze a simple case in steady state (Fig. 2).

In this example the reverberant field equation holds: 


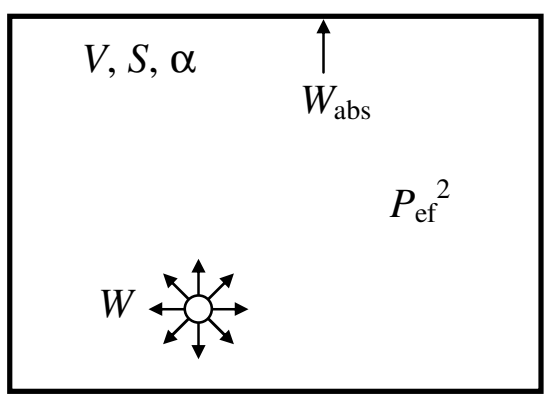

Fig. 2. A reverberant room in steady state with an acoustic power source $W$. The power $W_{\text {abs }}$ absorbed at the surfaces and the square effective pressure $P_{\text {ef }}{ }^{2}$ of the reverberant field are shown. $V$ is the volume, $S$, the surface, and $\alpha$, the absorption coefficient of the room.

$$
P_{\mathrm{ef}}^{2}=\rho_{\mathrm{o}} c \frac{4(1-\alpha)}{\alpha S} W
$$

where $\alpha$ is the mean sound absorption coefficient, $W$ the acoustic power, $S$ the inner area of the room and $\rho_{0} c$ the specific acoustic impedance of air [3].

While we could use $P_{\mathrm{ef}}^{2}$ as the analog of voltage, it is more convenient to use the acoustic energy density $D$, which is proportional to $P_{\mathrm{ef}}^{2}$ :

$$
D=\frac{P_{\mathrm{ef}}^{2}}{\rho_{\mathrm{o}} c^{2}} .
$$

This formula is valid for plane waves [3], but a diffuse field as we are assuming is the superposition of a large number of incoherent plane waves. Thus,

$$
D=\frac{4(1-\alpha)}{\alpha S c} W
$$

Given the analogy

$$
\begin{aligned}
& D \leftrightarrow V \\
& W \leftrightarrow I
\end{aligned}
$$

we can define an analog to electric resistance, which we shall call absorption resistance, as

$$
R_{\mathrm{abs}}=\frac{D}{W}=\frac{4(1-\alpha)}{\alpha S c} .
$$

Now we can draw the first circuit for the reverberant steady state (Fig.3).

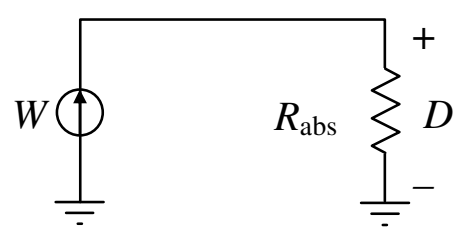

Fig. 3. Circuit of the reverberant steady state in a room.

In the previous example the air absorption, which is important for large rooms, was not taken into account. In this case (Fig. 4) $\alpha$ can be replaced by a coefficient $\alpha_{\text {tot }}$ given by

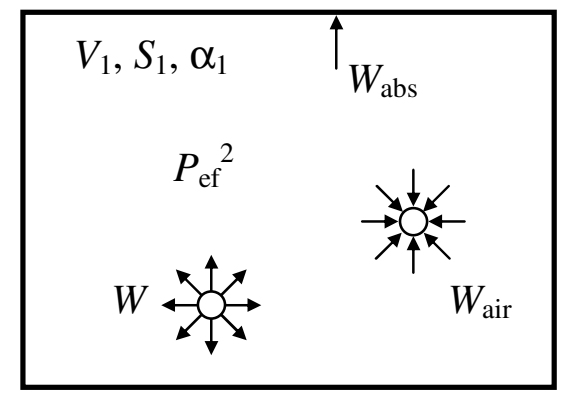

Fig. 4. A reverberant room in steady state, considering the acoustic power absorbed by the air.

$$
\alpha_{\text {tot }}=\alpha+\gamma_{\mathrm{m}} \frac{4 V}{S}
$$

where $V$ is the volume, $S$ the area and $\gamma_{\mathrm{m}}$, the coefficient of absorption in the air, in neper/m [3]. Then

$$
R=\frac{4\left(1-\alpha_{\text {tot }}\right)}{\alpha_{\text {tot }} S c} .
$$

It is interesting to note that this resistance can be considered as the parallel of two resistances, one of them representing the absorption by the surfaces, and the other, by the air.

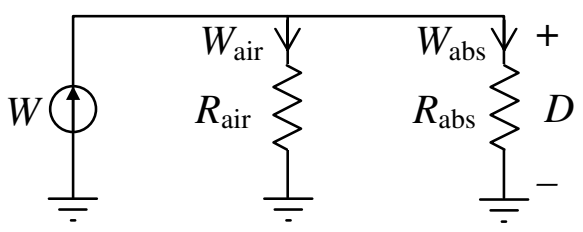

Fig. 5. Equivalent circuit for the steady state of the reverberant field in a room, including the absorption in the air.

Because of energy conservation, we have an equivalent of Kirchhoff's first law,

$$
\begin{gathered}
W_{\text {air }}=W-W_{\text {abs }} . \\
W_{\text {air }}=\left(\frac{\alpha_{\text {tot }}}{1-\alpha_{\text {tot }}}-\frac{\alpha}{1-\alpha}\right) \frac{S c}{4} D .
\end{gathered}
$$

We can look for a value $\alpha^{\prime}$ such that

$$
\frac{\alpha_{\text {tot }}}{1-\alpha_{\text {tot }}}-\frac{\alpha}{1-\alpha}=\frac{\alpha^{\prime}}{1-\alpha^{\prime}} .
$$

This value represents a hypothetical surface absorption equivalent to air absorption. Solving for $\alpha^{\prime}$

$$
\alpha^{\prime}=\frac{\gamma_{\mathrm{m}} \frac{4 V}{S}}{(1-\alpha)^{2}+\gamma_{\mathrm{m}} \frac{4 V}{S} \alpha} .
$$

The distinction between both absorption mechanisms is of conceptual interest and also illustrates a direct application of 
circuit theory to this model, but in practice we will work directly with

$$
R=R_{\text {air }} / / R_{\text {abs }}
$$

\section{COUPLED ROOMS IN STEADY STATE}

We shall next consider two rooms coupled by a small opening of area $S_{12}$ (Fig. 6).

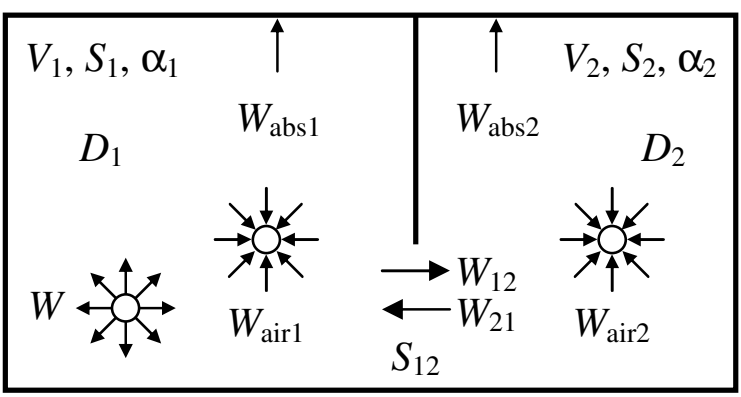

Fig. 6. Coupled rooms with surface and air absorption. In room 1 there is a sound source with acoustic power $W . S_{12}$ is the area of the opening connecting both rooms

The power loss in each room is

$$
\begin{aligned}
W_{\text {loss } 1} & =\frac{\alpha_{\mathrm{tot} 1} S_{1} c}{4\left(1-\alpha_{\mathrm{tot} 1}\right)} D_{1}, \\
W_{\text {loss } 2} & =\frac{\alpha_{\mathrm{tot} 2} S_{2} c}{4\left(1-\alpha_{\mathrm{tot} 2}\right)} D_{2} .
\end{aligned}
$$

On the other hand, the power arriving at each room through the opening is given by

$$
\begin{aligned}
& W_{12}=\frac{S_{12} c}{4} D_{1}, \\
& W_{21}=\frac{S_{12} c}{4} D_{2} .
\end{aligned}
$$

The following energy balance holds:

$$
\begin{aligned}
& W_{\text {loss } 1}+W_{12}-W_{21}=W \\
& W_{\text {loss } 2}+W_{21}-W_{12}=0
\end{aligned}
$$

Replacing equations (12) through (15) in (16) we get

$$
\begin{aligned}
& \left(\frac{\alpha_{\text {tot } 1} S_{1} c}{4\left(1-\alpha_{\text {tot } 1}\right)}+\frac{S_{12} c}{4}\right) D_{1}-\frac{S_{12} c}{4} D_{2}=W \\
& -\frac{S_{12} c}{4} D_{1}+\left(\frac{\alpha_{\text {tot } 2} S_{2} c}{4\left(1-\alpha_{\text {tot } 2}\right)}+\frac{S_{12} c}{4}\right) D_{2}=0
\end{aligned}
$$

If, by analogy with equation (6), we introduce the following circuit parameters

$$
\begin{gathered}
R_{1}=\frac{4\left(1-\alpha_{\mathrm{tot} 1}\right)}{\alpha_{\mathrm{tot} 1} S_{1} c}, \\
R_{2}=\frac{4\left(1-\alpha_{\mathrm{tot} 2}\right)}{\alpha_{\mathrm{tot} 2} S_{2} c}, \\
R_{12}=\frac{4}{S_{12} c},
\end{gathered}
$$

the system of equations (17) can be rewritten as

$$
\begin{aligned}
& \left(\frac{1}{R_{1}}+\frac{1}{R_{12}}\right) D_{1}-\frac{1}{R_{12}} D_{2}=W \\
& -\frac{1}{R_{12}} D_{1}+\left(\frac{1}{R_{2}}+\frac{1}{R_{12}}\right) D_{2}=0
\end{aligned}
$$

System (21) can be interpreted as the resolution of the circuit of Fig. 7 by the node potential method.

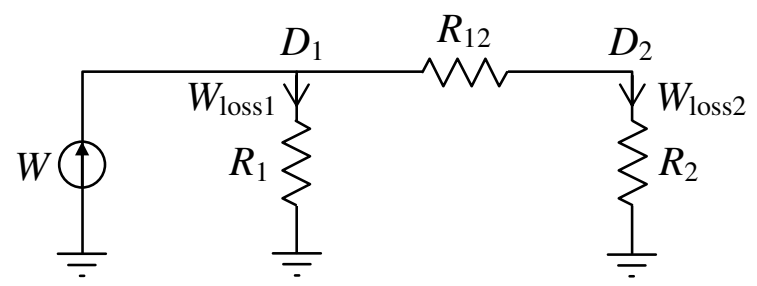

Fig. 7. Steady-state circuit model for two acoustically coupled rooms.

This system is easily solved, getting

$$
\begin{aligned}
& D_{1}=W \frac{R_{1}\left(R_{12}+R_{2}\right)}{R_{1}+R_{2}+R_{12}} \\
& D_{2}=W \frac{R_{1} R_{2}}{R_{1}+R_{2}+R_{12}}
\end{aligned}
$$

Circuit analysis allows to get interesting conclusions. For instance, from (20) we can see that the smaller the opening area the larger $R_{12}$, hence the weaker the coupling, Indeed, from equation (22) it is seen that the energy density in room 1 practically does not depend on the characteristics of room 2 (as $R_{12} \gg R_{2}$ ), while from (23) the energy density of the latter will be a small fraction ( $\left.\cong R_{2} / R_{12}\right)$ of the energy density in the former.

\section{ROOMS COUPLED BY A PARTITION}

We shall consider now two rooms coupled by a partition whose sound transmission coefficient ${ }^{1}$ is $\tau_{\mathrm{s}}$. In such case equations (14) and (15) will be affected by $\tau_{\mathrm{s}}$, i.e.,

$$
W_{12}=\tau_{\mathrm{s}} \frac{S_{12} c}{4} D_{1},
$$

1 The sound transmission coefficient is the ratio of the transmitted power to the incident power. 


$$
W_{21}=\tau_{\mathrm{s}} \frac{S_{12} c}{4} D_{2}
$$

from which

$$
R_{12}=\frac{4}{\tau_{\mathrm{s}} S_{12} c}
$$

Besides, it will be necessary to take into account the absorption coefficient of the partition, which will normally be less than 1, affecting also the mean absorption of both rooms.

An interesting example arises in the case of movable partition walls used in large rooms to accomplish different room distributions such as at conference and exhibition centers. Since in general $\tau_{\mathrm{s}}$ is relatively low, coupling is weak, but this is compensated because the common area is considerable.

Another typical situation is when there is communication through a suspended ceiling in cases in which a larger room has been partitioned to get several smaller spaces.

\section{SINGLE ROOM IN DYNAMIC CONDITIONS}

Transient behavior in a room is important at least in three situations. The first one is the onset of the sound field, the second, the sound decay typical of reverberation, and the third, the response to amplitude modulation (modulation transfer function), of interest for the analysis of speech intelligibility.

In order to analyze this problem we will start from the theoretical energy impulse response, which consists in an exponential decay

$$
D(t)=D_{\mathrm{o}} e^{\left(\ln (1-\alpha)-\gamma_{\mathrm{m}} \frac{4 V}{S}\right) \frac{c S}{4 V} t}
$$

This response is characterized by a time constant $\tau$ given by

$$
\tau=\frac{4 V}{c S} \frac{1}{-\ln (1-\alpha)+\gamma_{\mathrm{m}} \frac{4 V}{S}}
$$

In electric circuits this kind of response is associated with the discharge of a capacitor through a resistor, so we can propose a circuit component similar to a capacitor connected as shown in Fig. 8.

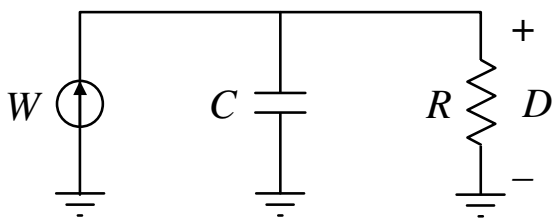

Fig. 8. Equivalent circuit for the reverberant state of a room.

In order that the time response be the same it is necessary that

$$
R C=\tau
$$

where $R$ is given by (6). Solving for $C$,

$$
C=\frac{\alpha_{\text {tot }}}{1-\alpha_{\text {tot }}-\ln (1-\alpha)+\gamma_{\mathrm{m}} \frac{4 V}{S}} .
$$

For $\alpha<<1$ we can approximate

$$
-\ln (1-\alpha) \cong \alpha
$$

so that

$$
C \cong \frac{V}{1-\alpha_{\text {tot }}}
$$

Sometimes, if $\alpha_{\text {tot }}<<1$, we can approximate

$$
C \cong V \text {. }
$$

As can be noted, the capacity is associated with the room volume, which is reasonable.

\section{COUPLED ROOMS IN DYNAMIC CONDITIONS}

Consider now two coupled rooms in non stationary state. Considering the time constant of each room as if they were not coupled, we can draw an equivalent circuit such as shown in Fig. 9.

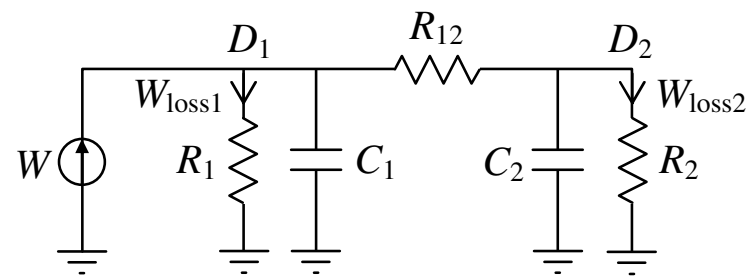

Fig. 9. Dynamic condition circuit model of two acoustically coupled rooms.

In this case, the node potential equations, written by simple inspection, are

$$
\begin{aligned}
& \left(\frac{1}{R_{1}}+C_{1} s+\frac{1}{R_{12}}\right) D_{1}-\frac{1}{R_{12}} D_{2}=W \\
& -\frac{1}{R_{12}} D_{1}+\left(\frac{1}{R_{2}}+C_{2} s+\frac{1}{R_{12}}\right) D_{2}=0
\end{aligned}
$$

Note that the equations have been written directly using the Laplace transform, avoiding the classic approach with differential equations (see, for example, [5], [6]) and taking advantage of the techniques that are commonplace in circuit analysis.

The solution of this system of equations is completely similar to the static case of equation (21). After some algebraic manipulation we get 


$$
\begin{gathered}
D_{1}=W \frac{R_{1} / / R_{12}\left(1+R_{2} / / R_{12} C_{2} s\right)}{1-\frac{R_{1} / / R_{12} R_{2} / / R_{12}}{R_{12}{ }^{2}}+\left(R_{1} / / R_{12} C_{1}+R_{2} / / R_{12} C_{2}\right) s+R_{1} / / R_{12} C_{1} R_{1} / / R_{12} C_{2} s^{2}} \\
D_{2}=W \frac{R_{1} / / R_{12} \frac{R_{2}}{R_{2}+R_{12}}}{1-\frac{R_{1} / / R_{12} R_{2} / / R_{12}}{R_{12}{ }^{2}}+\left(R_{1} / / R_{12} C_{1}+R_{2} / / R_{12} C_{2}\right) s+R_{1} / / R_{12} C_{1} R_{1} / / R_{12} C_{2} s^{2}}
\end{gathered}
$$

As we can see, it is a second-order system and it can be shown that its poles are real since it is a completely dissipative system. This means that there will be a double decay slope, which is characteristic of the reverberation of acoustically coupled spaces. The effect will be more noticeable in the receiving space, especially if the coupling is weak.

\section{CONCLUSION}

An approach based on circuit models has been presented for the analysis of acoustically coupled spaces. The model uses flow and force type analogies, where the flow variable is the acoustic power (the analog of the electric current) and the force variable is the volume energy density (the analog of voltage).

This approach allows to represent reverberant acoustic systems by means of a model that can be derived by simple inspection, in a similar fashion to what is customary in electric network analysis. The model is akin to the topology of the system.

Furthermore, the approach allows to profit from the vast collection of resources available for circuit resolution, a technique that has been developed for more than a century, including computer simulation.
Particularly interesting is the use of the Laplace transform, which allows to analyze the dynamics of coupled rooms, such as transient response and double or multiple reverberation slopes.

It is possible, for instance, to solve for the slopes of the energy impulse response. Of course, one should not be tempted to get the impulse response for its use in auralization since only the energy density is obtained. But it could be possible to simulate the late response applying the energy response to a suitably filtered random noise.

\section{REFERENCES}

[1] Tutte, W.T. Graph Theory. Cambridge University Press, 2001.

[2] Borutzky, Wolfgang (Editor), Bond Graph Modelling of Engineering Systems. Theory, Applications and Software Support. Springer, 2011.

[3] Beranek, Leo. Acoustics. ASA, 1986.

[4] Bergman, Theodore L.; Lavine, Adrienne S.; Incropera, Frank P.; Dewitt, David P. Fundamentals of Heat and Mass Transfer, 7th Ed., John Wiley \& Sons Inc., 2011.

[5] Cremer, Lothar; Müller, HA. Principles and Applications of Room Acoustics. Applied Science, London, 1982.

[6] Kuttruff, Heinrich. Room acoustics (5th edition). Spon Press, 2009 